\title{
Differences in the expression profiles of IncRNAs and mRNAs in partially injured anterior cruciate ligament and medial collateral ligament of rabbits
}

\author{
Huining Gu ${ }^{1}$, Siyuan Chen ${ }^{1}$, Mingzheng Zhang ${ }^{2}$, Yu Wen ${ }^{\text {Corresp., } 1}$, Bin Li ${ }^{\text {Corresp. } 2}$ \\ ${ }^{1}$ Department of Histology and Embryology, College of Basic Medical Sciences, China Medical University, Shenyang, China \\ 2 Department of Joint Surgery and Sports Medicine, Shengjing Hospital, China Medical University, Shenyang, China \\ Corresponding Authors: Yu Wen, Bin Li \\ Email address: ywen@cmu.edu.cn, surgeon_li@126.com
}

Long noncoding RNAs (IncRNAs), as a novel regulatory factor, are considered to play a vital role in various biological processes and diseases. However, the overall expression profile and biological functions of IncRNAs in the partially injured anterior cruciate ligament(ACL) and medial collateral ligament(MCL) have not been clearly explored. Partially injured models of ACL and MCL were established in 3-month-old healthy male New Zealand white rabbits. Expression of IncRNAs and mRNAs in the ligament tissue was detected by highthroughput sequencing technology, and biological functions of differentially expressed RNAs were evaluated by Gene Ontology(GO) and Kyoto Encyclopedia of Genes and Genomes(KEGG) analysis. Validation of several differentially expressed RNAs was performed using quantitative real-time PCR(qRT-PCR). Protein-protein interaction (PPI) analysis and competitive endogenous RNA (ceRNA) prediction were used to identify interactions among hub genes and the interaction among IncRNAs, miRNAs, and mRNAs. The results showed that compared with the normal group, there were 267 mRNAs and 329 IncRNAs differentially expressed in ACL and 726 mRNAs and 609 IncRNAs in MCL in the injured group. Compared with $\mathrm{MCL}, 420$ mRNAs and 470 IncRNAs were differentially expressed in ACL in the normal group; 162 mRNAs and 205 IncRNAs were differentially expressed in $A C L$ in the injured group. Several important IncRNAs and genes were identified, namely, COL7A1, LIF, FGFR2, EPHA2, CSF1, MMP2, MMP9, SOX5, LOX, MSTRG.1737.1, MSTRG.26038.25, MSTRG.20209.5, MSTRG.22764.1, and MSTRG.18113.1, which are closely related to inflammatory response, tissue damage repair, cell proliferation, differentiation, migration, and apoptosis. Further study of the functions of these genes may help to better understand the specific molecular mechanisms underlying the occurrence of endogenous repair disorders in $A C L$, which may provide new ideas for further exploration of effective means to promote endogenous repair of ACL injury. 
1 Differences in the expression profiles of IncRNAs and

2 mRNAs in partially injured anterior cruciate ligament

3 and medial collateral ligament of rabbits

4 Huining Gu ${ }^{1}$, Siyuan Chen ${ }^{1}$, Mingzheng Zhang ${ }^{2}, Y_{u}$ Wen ${ }^{1 *}$, Bin $\mathrm{Li}^{2}$ *

$5{ }^{1}$ Department of Histology and Embryology, College of Basic Medical Sciences, China Medical

6 University, Shenyang ,Liaoning, China

$7 \quad{ }^{2}$ Department of Joint Surgery and Sports Medicine, Shengjing Hospital, China Medical

8 University, Shenyang,Liaoning, China

9 Corresponding Author:

$10 \mathrm{Yu} \mathrm{Wen}{ }^{1}$, Bin $\mathrm{Li}^{2}$

No.77 Puhe Road,Shenyang North New Area, Shenyang, Liaoning Province, 110122, China

\section{Abstract}

Long noncoding RNAs (lncRNAs), as a novel regulatory factor, are considered to play a vital role in various biological processes and diseases. However, the overall expression profile and biological functions of lncRNAs in the partially injured anterior cruciate ligament(ACL) and medial collateral ligament(MCL) have not been clearly explored. Partially injured models of ACL and MCL were established in 3-month-old healthy male New Zealand white rabbits. Expression of lncRNAs and mRNAs in the ligament tissue was detected by high-throughput sequencing technology, and biological functions of differentially expressed RNAs were evaluated by Gene Ontology(GO) and Kyoto Encyclopedia of Genes and Genomes(KEGG) analysis. Validation of several differentially expressed RNAs was performed using quantitative real-time PCR(qRTPCR). Protein-protein interaction (PPI) analysis and competitive endogenous RNA (ceRNA) prediction were used to identify interactions among hub genes and the interaction among lncRNAs, miRNAs, and mRNAs. The results showed that compared with the normal group, there were 267 mRNAs and 329 lncRNAs differentially expressed in ACL and 726 mRNAs and 609 lncRNAs in MCL in the injured group. Compared with MCL, 420 mRNAs and 470 lncRNAs were differentially expressed in ACL in the normal group; 162 mRNAs and 205 lncRNAs were differentially expressed in ACL in the injured group. Several important lncRNAs and genes were identified, namely, COL7A1, LIF, FGFR2, EPHA2, CSF1, MMP2, MMP9, SOX5, LOX, 
32 which are closely related to inflammatory response, tissue damage repair, cell proliferation, differentiation, migration, and apoptosis. Further study of the functions of these genes may help to better understand the specific molecular mechanisms underlying the occurrence of endogenous repair disorders in ACL, which may provide new ideas for further exploration of effective means to promote endogenous repair of ACL injury.

\section{Introduction}

ACL is a connective tissue composed of bundles of collagen fibers, connects the femur and tibia, which prevents excessive anterior translation and internal rotation of the tibia with respect to the femur and provides mechanical stability to the knee joint(Wang, et al.,2020). MCL is the main stabilizing structure of the medial side of the knee, which resists valgus stress, provides static and dynamic stability, and assists in resisting rotational stress and anterior-posterior translation(Guo, et al.,2019). Ninety percent of knee ligament injuries involve ACL or MCL, and present a commonly encountered problem in modern sports medicine(Georgiev, et al.,2018). ACL injury is the leading cause of recurrent knee instability and may result in pain, limited range of movement, muscle weakness, biomechanical changes, and reduced physical activity level, while potentially affecting the function of other knee structures such as meniscal tears and articular cartilage degeneration, imposing a significant financial burden on the health care system(Jia, et al.,2017; Wang, et al.,2020) .

The self-healing response after ACL injury is very poor. Currently, the clinical treatment regarding ACL injury is mainly surgical repair or reconstruction. However, even after surgery, the ACL still cannot regain its normal biological functionality(Xie, et al.,2013, 2014; Cai, et al.,2017). Recent evidence also indicates that postoperative inflammation may damage synovial stem cells and lead to an impaired joint environment, which inhibits tissue healing(Wang, et al.,2020), and even some patients may develop complications after surgery such as joint stiffness and osteoarthritis (OA)(Xie, et al.,2013, 2014; Cai, et al.,2017). In contrast, injured MCL have a relatively better ability to self-heal and, in some cases, fully recover their function(Furumatsu, et al.,2010; Xie, et al.,2013).

In recent years, scholars have proposed several possible explanations for the differences between the healing abilities of ACL and MCL after injury, including the different ultrastructural characteristics of connective tissue cells in ACL and MCL(Lyon, et al.,1991), the differences in 
62 cellular properties and responses to growth factors between ACL and MCL(Yoshida and

63 Fujii,1999), MCL's improved capability to increase blood supply through increased angiogenesis

64 and blood flow(Bray, et al.,2003); the different properties of ACL and MCL stem cells(Zhang, et

65 al.,2011); the differential expression of the LOX family, MMP-2, MMP-9, type I and V collagen

66 and type III procollagen in ACL and MCL fibroblasts(Xie, et al.,2013; Georgiev, et al.,2018,

67 2019). However, the exact molecular mechanisms responsible for the endogenous repair

68 difference of ACL and MCL are still unclear.

LncRNAs are a class of heterologous transcripts defined by sequence lengths exceeding 200 nucleotides and lacking any apparent protein-coding potential(Kopp,2019). As a novel regulator, lncRNAs play key roles in biological processes such as cell proliferation, differentiation, apoptosis, autophagy, inflammation, and angiogenesis, as well as epigenetic, transcriptional, and post-transcriptional modifications(Mousavi, et al.,2013; Toiyama, et al.,2014), regulating the expression and function of protein-coding genes and participating in the pathogenesis of various types of diseases(Gan, et al.,2020). In addition, the role of some lncRNAs in the inflammatory response, proliferation and apoptosis of certain human ligament cells has been demonstrated(Wang, et al.,2021; Zhou, et al.,2021). Based on the above findings, we speculate that there may be differentially expressed lncRNAs involved in the regulation of genes related to ligament injury repair in ACL and MCL, resulting in the difference in healing ability between the two. However, the role of lncRNAs in knee ligament injury remains unclear, and there has been no exploration of the expression profiles, biological functions, or signaling pathways of lncRNAs in partially injured ACL and MCL. Therefore, we developed a partial injury model for rabbit ACL and MCL and examined the expression of lncRNAs and mRNAs in normal and partially injured ACL and MCL of rabbits by high-throughput sequencing. GO and KEGG enrichment analyses were performed to explore the potential biological functions and pathways of action of differentially expressed genes(DEGs). Additionally, PPI analysis and ceRNA co-expression network were used to identify hub genes and the interaction among lncRNAs, mRNAs, and miRNAs. We hope these results can provide a theoretical basis for exploring the possible molecular mechanism of endogenous repair disorder after ACL injury.

\section{Materials \& Methods}

\section{Animals and partially injured models of ACL and MCL}


92 3-month-old healthy male New Zealand white rabbits $(\mathrm{n}=6)$ were purchased from Qingdao

93 Kangda Biological Technology Co, Ltd. (Qingdao, China).Rabbits were bred at the Department 94 of Experimental Animals of China Medical University(Shenyang, China) at $23 \pm 2^{\circ} \mathrm{C}$ with 12 -

95 hour light-dark cycles. The Medical Ethics Committee China Medical University approved the 96 animal experiments on October 22, 2020 (approval No. CMU2020310).

Animals were randomly divided into two groups with 3 rabbits in each group. Rabbits in the control group were bred under normal conditions and labeled N1, N2, and N3 respectively. The following treatment was done for the injured group : (1) Surgery to establish bilateral partially injured models of ACL and MCL: A longitudinal incision was made on the medial side of the knee joint of the rabbit, with an incision length of approximately $1.5 \mathrm{~cm}$. The skin was incised layer by layer, and the subcutaneous tissue and joint capsule were bluntly separated to fully expose the ACL and MCL. ACL and MCL were laterally punctured by a $20 \mathrm{ml}$ needle at the proximal $1 / 3$ of the femur and subsequently lacerated by a needle, resulting in blunt laceration of most(approximately2/3) of the ligament. The wounds were cleaned with physiological saline and the joint capsule and skin were sutured. (2)Postoperatively, ceftriaxone sodium $75 \mathrm{mg} / \mathrm{kg}$ was administered intramuscularly once daily for 3 days to combat the infection. Rabbits in the injured group were labeled as I1, I2, and I3. Rabbits in both groups were sacrificed to collect ligaments after deep anesthesia at 1week after modeling. For all ligaments, snap-frozen in liquid nitrogen within $15 \mathrm{~min}$ of surgery and stored at $-80^{\circ} \mathrm{C}$. 2 normal ACLs and MCLs and 2 injured ACLs and MCLs were randomly selected for RNA-Seq, and the rest of ligaments were used for qRT-PCR.

\section{RNA extraction, library construction, and RNA-Seq}

Total RNA of each sample was extracted from the ligament tissue as per the instruction manual of the TRlzol Reagent (Life technologies, California, USA). RNA concentration was checked using Nanodrop2000 (Thermo Fisher Scientific, Waltham,USA), and RNA integrity was checked using Agient2100 (Agilent Technologies Inc., Santa Clara, CA, USA) and LabChip GX(PerkinElmer Inc.,Waltham,USA). RNA contamination was monitored on agarose gels. The cDNA libraries were constructed using the Ribo-off rRNA Depletion Kit after the samples passed the quality control, and then sequenced using Illumina NovaSeq 6000 platform. 
123 calculate FPKMs of both lncRNAs and coding genes in each sample after completion of the

124 comparison analysis. Differential expression analysis of two groups was performed using

125 normalized count data which were derived using the DESeq2 package. Fold Change (FC)

126 indicates the ratio of expression between two groups, and False Discovery Rate (FDR) is

127 obtained by correcting the p-value for the significance of the difference (p-value) using

128 Benjamini and Hochberg's approach. Genes with FDR $<0.01$ and the absolute value of FC $\geq 2$

129 were assigned as differentially expressed.

130 Gene functional annotation

131 Gene function was annotated based on the following databases:Nr (NCBI non-redundant 132 protein sequences); Pfam (Protein family); KOG/COG (Clusters of Orthologous Groups of 133 proteins); Swiss-Prot (A manually annotated and reviewed protein sequence database); KEGG 134 (Kyoto Encyclopedia of Genes and Genomes); GO (Gene Ontology). GO enrichment analysis 135 was performed using the topGO R package, and Kobas software was used to test the statistical 136 enrichment in the KEGG pathway.

137

138 139

\section{Construction of PPI Network}

The sequences of the DEGs was blast (blastx) to the genome of a related species (the protein protein interaction of which exists in the STRING database:http://string-db.org/) to get the predicted PPI of these DEGs. Cytoscape software (version 3.8.2; https://cytoscape.org) was then used to build a PPI network and identify hub genes.

\section{Construction of IncRNA-miRNA-mRNA Network}

Competitive endogenous RNA (ceRNA) (Salmena, et al.,2011)has gained attention in recent years as a new mode of transcriptional regulation. LncRNAs can be used as a miRNA sponge to compete with miRNAs and inhibit the regulation of miRNAs on the target gene, thus indirectly regulating gene expression(Xiao, et al.,2019). Our study obtains candidate ceRNA relationship pairs by targeting relationships of miRNAs that satisfy the following conditions. (1) the number of identical miRNAs between ceRNAs should be greater than 5. (2) the p-value of the hypergeometric test is less than 0.01, and the corrected FDR value is less than 0.01. (3) The results of co-expression analysis are taken into account to obtain a ceRNA co-expression network where both ceRNAs are co-expressed with each other. Based on the ceRNA network, the relationship pairs that differed in all three for each differential combination were extracted and 
153 visualized using Cytoscape software. The top 50 nodes in each group were identified using the

154 cytoHubba plug-in in Cytoscape and Degree method mapped with Cytoscape.

\section{Quantitative real-time PCR}

156 Total RNA of each sample was extracted from the ligament tissue according to the 157 instruction manual of the TRlzol Reagent (Ambion, Massachusetts, USA). The PrimeScript II 1st 158 Strand cDNA Synthesis Kit (Takara Bio inc., Kusatsu, Japan) and SYBR Premix ex Taq II 159 (Takara) were used for reverse transcription and quantitative real-time PCR (qRT-PCR) assay. 160 Polymerase chain reaction primer sequences are listed in Supplementary Table 1(Sangon Biotech 161 Co., Ltd., Shanghai, China). $\beta$-Actin was applied as an internal control. For quantitative analysis 162 of differential expression, data were processed by the $2^{-\Delta \Delta \mathrm{Ct}}$ method and the expression of all 163 lncRNAs and mRNAs were presented as relative fold changes to $\beta$-actin, which was repeated 164 three times for each sample.

\section{Statistical analysis}

166 All statistical data are expressed as the mean \pm standard error of mean (SEM). Differences 167 were analyzed by t-test using Graphpad Prism 8 statistical software. P-value $<0.05$ was 168 considered statistically significant.

\section{Data Availability Statement}

All sequences are accessible via SRA using the accession PRJNA751479. Data is available at NCBI SRA, accession numbers: SRR15858594 to SRR15858601.

\section{Results}

\section{Statistical results of RNA sequencing data}

174 The statistical results of RNA-seq data are shown in Supplementary Table 2. 31217 genes were detected, and 10903 new genes were discovered, 2466 of which were functionally annotated. On the basis of the comparison results, gene expression analysis was carried out. Based on the expression of genes in different samples, 1233 DEGs were identified, and functional annotation and enrichment analysis were performed. A total of 26242 lncRNAs and 1263 DELs were identified, and the obtained lncRNAs were divided into four groups with the following classification and percentage (Supplementary Figure 1): lincRNA (40\%), 
182

183

\section{DEGs screening, gene ontology, and Kyoto encyclopedia of genes and genomes pathway} analysis.

\section{Differences between normal and injured groups}

In total, 267 mRNAs were differentially expressed in the injured ACL compared with normal controls, of which 165 were up-regulated, and 102 were down-regulated(Figure1A and B). DEGs were enriched for several GO pathways such as cellular adhesion, positive regulation of angiogenesis, cellular response to tumor necrosis factor, elastic fiber assembly, extracellular exosomes, proteinaceous extracellular matrix, extracellular matrix, cellular adhesion molecule binding, and fibroblast growth factor binding(Figure1C to 1E). The KEGG pathway enriched analysis results showed that DEGs were mainly enriched in PI3K-Akt signaling pathway, Cell cycle, PPAR signaling pathway, ECM-receptor interaction, Cellular senescence, AMPK signaling pathway, p53 signaling pathway, Ferroptosis, TNF signaling pathway, and IL-17 signaling pathway, which were related to cell proliferation and apoptosis or inflammation(Figure1F).

726 mRNAs were differentially expressed in the injured MCL compared with normal controls, of which 372 were up-regulated, and 354 were down-regulated(Figure2A and B). DEGs were enriched for several GO pathways such as positive regulation of ERK1 and ERK2 cascade, inflammatory response, acute-phase response, blood vessel remodeling, phagocytosis, engulfment, positive regulation of phagocytosis, leucocyte migration involved in the inflammatory response, extracellular exosomes, cell surface, proteinaceous extracellular matrix, lysosome, extracellular matrix, cellular adhesion molecule binding, collagen binding, cytokine receptor activity, and collagen receptor activity(Figure2C to 2E). The KEGG pathway enriched analysis results showed that DEGs were mainly enriched in Cytokine-cytokine receptor interaction, Cell adhesion molecules, and Phagosome, which were related to cell proliferation and apoptosis(Figure2F).

\section{Differences between the ACL and MCL groups}

In the normal group, 420 mRNAs were differentially expressed in ACL compared to MCL, of which 178 were up-regulated and 242 were down-regulated(Figure3A and B). DEGs were enriched for several GO pathways such as extracellular matrix organization, cell adhesion, positive regulation of ERK1 and ERK2 cascade, blood vessel development, regulation of fibroblast growth factor receptor signaling pathway, extracellular exosome, proteinaceous extracellular matrix, cell surface, extracellular matrix, collagen type I trimer, collagen type IX 
213 trimer, collagen type II trimer, extracellular matrix structural constituent, growth factor activity,

214 cell adhesion molecule binding, platelet-derived growth factor binding, and extracellular matrix

215 binding(Figure3C to 3E). The KEGG pathway enriched analysis results showed that DEGs were

216 mainly enriched in PI3K-Akt signaling pathway, Focal adhesion, ECM-receptor interaction, Cell

217 adhesion molecules, ErbB signaling pathway, FcyR-mediated phagocytosis, Leukocyte

218 transendothelial migration, and TNF signaling pathway, which were related to cell proliferation,

219 differentiation, migration apoptosis, and inflammation(Figure3F).

220 In the injured group, 162 mRNAs were differentially expressed in ACL compared with 221 MCL, of which 82 were up-regulated and 80 were down-regulated(Figure4A and B). DEGs were 222 enriched for several GO pathways such as elastic fiber assembly, positive regulation of 223 mesenchymal stem cell differentiation, proteinaceous extracellular matrix, cell surface, elastic 224 fiber, collagen type II trimer, and extracellular matrix structural constituent(Figure4C to 4E). The 225 KEGG pathway enriched analysis results showed that DEGs were mainly enriched in ECM226 receptor interaction, Focal adhesion, PI3K-Akt signaling pathway, and Cell adhesion molecules, 227 which were related to cell proliferation and apoptosis(Figure4F).

DELs screening, gene ontology, and Kyoto encyclopedia of genes and genomes pathway analysis.

\section{Differences between normal and injured groups}

In total, 329 lncRNAs were differentially expressed in the injured ACL compared with normal controls, of which 209 were up-regulated and 120 were down-regulated(Supplementary Figure2A and B). Target genes of DELs were enriched for several GO pathways such as positive regulation of angiogenesis, positive regulation of inflammatory response, proteinaceous extracellular matrix, extracellular matrix, growth factor activity, chemokine activity, and cell adhesion molecule binding(Supplementary Figure2C to 2E). The KEGG pathway enriched analysis results showed that target genes of DELs were mainly enriched in Cytokine-cytokine receptor interaction, cAMP signaling pathway, cGMP-PKG signaling pathway, Chemokine signaling pathway, JAK-STAT signaling pathway, and ErbB signaling pathway, which were associated with cell proliferation, differentiation, and migration apoptosis(Supplementary

241 Figure2F).

242609 lncRNAs were differentially expressed in the injured MCL compared with normal 243 controls, of which 263 were up-regulated, and 346 were down-regulated(Supplementary 
244 Figure3A and B). Target genes of DELs were enriched for several GO pathways such as positive 245 regulation of cell proliferation, cell division, positive regulation of angiogenesis, growth, platelet246 derived growth factor receptor signaling pathway, extracellular exosomes, focal adhesion, 247 proteinaceous extracellular matrix, growth factor activity, and cell adhesion molecule 248 binding(Supplementary Figure3C to 3E). The KEGG pathway enriched analysis results showed 249 that target genes of DELs were mainly enriched in PI3K-Akt signaling pathway, cAMP signaling 250 pathway, Focal adhesion, Cell adhesion molecules, ECM-receptor interaction, and ErbB signaling 251 pathway, which were associated with cell proliferation, differentiation, and migration 252 apoptosis(Supplementary Figure3F).

\section{Differences between the ACL and MCL groups}

254 In the normal group, 470 lncRNAs were differentially expressed in ACL compared to MCL, 255 of which 166 were up-regulated and 304 were down-regulated(Supplementary Figure4A and B).

256 Target genes of DELs were enriched for several GO pathways such as positive regulation of 257 ERK1 and ERK2 cascade, positive regulation of JNK cascade, regulation of cellular metabolic 258 process, extracellular exosome, focal adhesion, proteinaceous extracellular matrix, stress fiber, 259 growth factor activity, and cell adhesion molecule binding(Supplementary Figure4C to 4E). The 260 KEGG pathway enriched analysis results showed that target genes of DELs were mainly enriched 261 in PI3K-Akt signaling pathway, MAPK signaling pathway, Focal adhesion, cGMP-PKG 262 signaling pathway, ECM-receptor interaction, and Ferroptosis, which were associated with cell 263 proliferation, differentiation, and migration apoptosis(Supplementary Figure4F).

264 In the injured group, 205 lncRNAs were differentially expressed in ACL compared to MCL, 265 of which 116 were up-regulated and 89 were down-regulated(Supplementary Figure5A and B). 266 Target genes of DELs were enriched for several GO pathways such as acute-phase response, 267 regulation of ERK1 and ERK2 cascade, and proteinaceous extracellular matrix(Supplementary 268 Figure5C to 5E). The KEGG pathway enriched analysis results showed that target genes of DELs 269 were mainly enriched in cAMP signaling pathway, Focal adhesion, cGMP-PKG signaling 270 pathway, ECM-receptor interaction, and ErbB signaling pathway, which were associated with cell 271 proliferation, differentiation, and migration apoptosis(Supplementary Figure5F).

\section{Identifying candidate genes for PCR-validation}

273 DEGs related to the altered pathway were further searched in the PubMed literature 274 database. "Gene symbol ”(gene name) was used as a query keyword and searched in the“ 
275 title/abstract ". 3 randomly selected up- and down-regulated genes associated with tissue damage

276 repair were validated in each group. Finally, since very little information was available on DELs,

2773 randomly selected DELs in each of the top 5 up-regulated and top five down-regulated DELs

278 were chosen for further validation (Figure 5). All the results of qRT-PCR are corresponded to

279 RNA-seq data.

\section{Construction of IncRNA-miRNA-mRNA Network}

281 Based on the ceRNA regulatory network, the top 50 nodes in each group (Figures 6A to 6D) 282 were identified, including lncRNAs, miRNAs, and mRNAs(only 45 nodes in the normal group of 283 ACL compared to the injured group). Each DEGs could be associated with one or more miRNAs 284 and lncRNAs, which contained some genes related to the tissue damage repair process, such as 285 COL7A1, LIF, FGD5, SLC20A1, and their associated lncRNAs and miRNAs were searched for, 286 thus their networks were displayed with Cytoscape (Figures 6E to 6G). The ceRNA network 287 suggested that lncRNAs could not only participate in the regulation of biological processes 288 independently but also act as miRNA sponges together to affect downstream gene expression and 289 thus participate in the processes after ACL and MCL injury.

\section{Construction of PPI Network}

We used PPI analysis and online STRING platform to detect the interaction effects between proteins in each group of DEGs, and then using cytoHubba plug-in and MCC method, we identified the top 30 ranked hub genes in each group (Figures 7A to 7D), which contained several genes related to the tissue damage repair process, such as FGFR2, HBEGF, EPHA2, CSF1, MMP2, MMP9, SOX5, LOX.

Discussion

In this study, we simultaneously detected mRNAs and lncRNAs in normal and partially injured ACL and MCL of rabbits for the first time and provide a new gene resource for studying the role of lncRNAs in the development of partial injury in ACL and MCL. In addition, we established a ceRNA network and further analyzed DEGs using PPI to explore the specific molecular mechanism of endogenous repair disorder in ACL.

Ligaments are similar to tendons belonging to fibrous connective tissue, mainly composed of fibroblasts and extracellular matrix. The main component of the extracellular matrix of ligaments is neatly arranged type I collagen fibers, in addition to small amounts of other 
305 components such as elastin, decorin, dimeric glycans, and fibronectin(Leong, et al.,2020). The 306 repair of ligaments injuries is accomplished through a typical wound healing pathway that 307 involves three main phases: inflammation, proliferation, and remodeling involving various cell 308 types, cytokines, and extracellular matrix factors, and is closely related to inflammation, 309 angiogenesis, the proliferation of fibroblasts, remodeling of the extracellular matrix, and the conversion of type III to type I collagen(Cottrell, et al.,2016; Lipman, et al.,2018). Our study showed that there were significant differences between ACL and MCL in terms of cellular composition, sensitivity to inflammatory responses, and cell proliferation and metabolic processes, which may be responsible for the differences in their healing capacity. collagen receptor activity are specifically exhibited in the MCL after injury. Previous studies have shown that injured MCL has a better ability to increase blood supply relative to injured ACL(Bray, et al.,2003). Adequate blood supply is necessary for ligament healing, and alterations in peripheral vascular structures after acute injury can also promote hematoma for hemostasis purposes(Lipman, et al.,2018). The enrichment of vascular remodeling in the injured MCL in GO analysis may account for its ability to have a better blood supply, which may have contributed to the different healing abilities of the ACL and MCL after injury. The main extracellular matrix component of ligaments is collagen, and the process of remodeling in the late stages of ligament injury is closely related to the remodeling of the extracellular matrix and the conversion of type III collagen to type I collagen(Cottrell, et al.,2016; Lipman, et al.,2018). The enrichment of collagen-binding domains and collagen receptor activity in the injured MCL in GO analysis may be more favorable for ligament repair in the late stages of injury. In addition, KEGG analysis showed that DEGs and the target genes of DELs of injured ACL appeared enriched in IL-17 signaling pathway, Cellular senescence, p53 signaling pathway, Ferroptosis, JAK-STAT signaling pathway. As a pro-inflammatory cytokine, IL-17 exerts its effects by promoting the expression of matrix metalloproteinases and proinflammatory genes, which can result in neutrophil infiltration and inflammation in tissue(Iwakura, et al.,2011). Ferroptosis is an iron-dependent, novel form of programmed cell death accompanied by massive iron accumulation and lipid peroxidation(Li, et al.,2020). P53 signaling pathway regulates a variety of cellular processes, including apoptosis, 334 growth inhibition, cell cycle progression inhibition, cellular senescence after stress, autophagy, 335 and apoptosis(Jeffries and Krupenko,2018). JAK-STAT signaling pathway is a signal 
336 transduction pathway stimulated by cytokines involved in many critical biological processes such

337 as cell proliferation, differentiation, apoptosis, and immune regulation(Xin, et al.,2020). Studies

338 have shown that regulated inflammation largely facilitates tissue repair, while excessive or 339 persistent inflammation may lead to poor tissue repair outcomes(Thomopoulos, et al.,2015). The 340 enrichment of the above pathways seen in the injured ACL may indicate that there may be a more 341 severe inflammatory response after ACL injury and that more cell death pathways are activated, 342 resulting in a poorer healing capacity.

343 Subsequently, we established a ceRNA network and further analyzed it, obtaining some 344 important nodes such as COL7A1, LIF, FGD5, SLC20A1, and the regulatory network associated 345 with them. COL7A1 plays a key role in physiological wound healing, supporting dermal 346 fibroblast migration and regulating their cytokine production in granulation tissue, contributing to 347 skin wound healing, loss of COL7A1 harms both the process and outcome of skin 348 healing(Nyström, et al.,2013). LIF is a glycoprotein in the interleukin-6 cytokine family, which 349 has pleiotropic actions throughout the body, affecting stem cell self-renewal, cell proliferation, 350 differentiation and survival(Metcalf,2003). Recent studies have shown that in human myogenic 351 cells, LIF can increase the number of myogenic cells by increasing mitosis and decreasing 352 apoptosis(Broholm, et al.,2012) and that LIF has neurodegenerative and protective functions in 353 perinatal hypoxic-ischemic brain injury(Lin, et al.,2020) and has some pro-angiogenic 354 potential(Santos, et al.,2020). Our results showed a trend of upregulation of both COL7A1 and 355 LIF in ACL and MCL after injury, perhaps with positive effects on healing after injury in both, 356 but with differences in their degree of change and regulatory networks. In addition, FGD5 can 357 regulate the pro-angiogenic effect of VEGF in vascular endothelial cells(Kurogane, et al.,2012), 358 which showed a trend of down-regulation in ACL after injury, which might also have an impact 359 on the recovery of hemopoietic capacity after ACL injury. SLC20A1 may promote the production 360 of pro-inflammatory and chemotactic mediators and ROS(Koumakis, et al.,2019), and in a 361 comparison of normal group ACL with MCL, SLC20A1 was found to be significantly 362 upregulated in ACL, and such differences may make ACL more prone to more inflammatory 363 responses. Meanwhile, we have obtained several important lncRNAs associated with the 364 regulation of these genes on the basis of the ceRNA network, including MSTRG.1737.1, 365 MSTRG.26038.25, MSTRG.20209.5, MSTRG.22764.1, and MSTRG.18113.1. These lncRNAs 366 might serve as non-coding gene candidates for further study of the pathogenesis of endogenous 
367 repair disorders in ACL. Elucidation of their interactions with the corresponding candidate genes

368 would help to better understand the molecular mechanisms by which lncRNAs regulate the 369 occurrence of endogenous repair disorders in ACL.

370 We additionally used PPI analysis to predict the interaction effects between the proteins of 371 each group of DEGs, and then obtained some hub genes such as FGFR2, EPHA2, CSF1, MMP2, 372 MMP9, SOX5, LOX. FGFR2b is involved in maintaining the stability of the internal skin 373 environment and wound healing(Katoh,2009), and previous studies on ligament healing have also 374 shown that FGFR2 plays a role in the proliferation of injured ligament fibroblasts and endothelial 375 cells(Rösler,1979). EPHA2 has been reported as an important factor in regulating cell 376 permeability and tight junctions in brain endothelial cells, and the inactivation of EPHA2 377 promotes tight junction formation and impairs angiogenesis in brain endothelial cells(Zhou, et 378 al.,2011). EPHA2/Ephrin-A1 signaling complexes restrict the migration of corneal epithelial 379 cells(Kaplan, et al.,2012). Recent studies have shown that the EPHA2 signaling pathway is involved in the permeability and inflammatory response to lipopolysaccharide-induced lung injury, and the application of EPHA2 monoclonal antibody to inhibit the expression of EPHA2 can reduce lung injury(Hong, et al.,2016). CSF1 influences the environment of renal epithelial 383 cell growth, proliferation, and differentiation, and promotes matrix remodeling and cell replacement during inflammation, contributing to kidney growth and endogenous repair following injury(Alikhan, et al.,2011). SOX5 acts as a transcription factor involved in regulating embryonic development and determining cell fate(Chen, et al.,2018), as well as in regulating differentiation, proliferation and neuronal development. Recent studies suggest the neuroprotective effects of SOX5 against ischemic stroke by regulating VEGF/PI3K/AKT pathway(Zhang, et al.,2021). MMP2 and MMP9 degrade extracellular matrix (ECM) proteins, such as type IV and V collagen, elastin, and vitreous junction proteins, and play key roles in connective tissue remodeling, demyelination and remyelination after injury, inflammation and glial cell reactivity, cell genesis and migration, and angiogenesis(Verslegers, et al.,2013; Xie, et al.,2018) Recent studies have found that MMP9 mediates the changes in collagen fibril organization that occur in acute and chronic tissue injury and has a regulatory effect on collagen structure after trauma(LeBert, et al.,2015). LOX is a copper-dependent amine oxidase that plays a key role in matrix synthesis by catalyzing the formation of crosslinks between collagen and elastin fibers(Lucero and Kagan,2006). In recent years, studies on the differential healing ability 
398 of injured ACL and MCL have also suggested possible explanations for the differential 399 expression of MMPs and LOX on several occasions(Xie, et al.,2013; Georgiev, et al.,2018). 400 Several previous studies have demonstrated that imbalance in ECM biosynthesis and degradation 401 caused by altered LOX and MMP expression may be responsible for the defective ACL 402 repair(Beye, et al.,2008; Xie, et al.,2013; Zhang, et al.,2017; Wang, et al.,2020) which correspond 403 to our analysis of the RNA-seq data. Further exploration of the role of these genes after ACL 404 injury may be more beneficial to our understanding and search for specific molecular 405 mechanisms of endogenous repair disorders in ACL.

406 Our study has some limitations. Experimental validation of the putative ACL endogenous 407 repair disorder-related genes and lncRNAs warrants further investigation. The small 408 reproducibility of the samples may result in incorrect screening of specific lncRNAs and genes, 409 and further studies will verify their reproducibility and reliability. Similarly, efforts should be 410 made to determine the specific roles of other DEGs, and DELs identified in this study and to 411 place them in the larger context of the progression of endogenous repair disorders in ACL.

\section{Conclusions}

413 In conclusion, our study provides the first preliminary analysis of lncRNAs and mRNAs 414 differentially expressed in normal and injured groups of ACL and MCL and predicts several 415 important lncRNAs and genes, which may serve as gene candidates for further study of the 416 pathogenesis of endogenous repair disorders in ACL. Further study of the functions of these 417 genes and their interactions with lncRNAs may help to better understand the specific molecular 418 mechanisms underlying the occurrence of endogenous repair disorders in ACL, which may 419 provide new ideas for further exploration of effective means to promote endogenous repair of 420 ACL injury.

\section{References}

Alikhan MA, Jones CV, Williams TM, et al. 2011. Colony-stimulating factor-1 promotes kidney growth and repair via alteration of macrophage responses. Am J Pathol. 179(3): 1243-56.

Beye JA, Hart DA, Bray RC, McDougall JJ, Salo PT. 2008. Injury-induced changes in mRNA levels differ widely between anterior cruciate ligament and medial collateral ligament. Am J Sports Med. 36(7): 1337-46.

Bray RC, Leonard CA, Salo PT. 2003. Correlation of healing capacity with vascular response in the anterior cruciate and medial collateral ligaments of the rabbit. J Orthop Res. 21(6): 1118-23. 
Broholm C, Brandt C, Schultz NS, Nielsen AR, Pedersen BK, Scheele C. 2012. Deficient leukemia inhibitory factor signaling in muscle precursor cells from patients with type 2 diabetes. Am J Physiol Endocrinol Metab. 303(2): E283-92.

Cai L, An S, Liao J, et al. 2017. Influences of Tumor Necrosis Factor- $\alpha$ on Lysyl Oxidases and Matrix Metalloproteinases of Injured Anterior Cruciate Ligament and Medial Collateral Ligament Fibroblasts. J Knee Surg. 30(1): 78-87.

Chen X, Zheng Q, Li W, et al. 2018. SOX5 induces lung adenocarcinoma angiogenesis by inducing the expression of VEGF through STAT3 signaling. Onco Targets Ther. 11: 5733-5741.

Cottrell JA, Turner JC, Arinzeh TL, O\&\#39, Connor JP. 2016. The Biology of Bone and Ligament Healing. Foot Ankle Clin. 21(4): 739-761.

Furumatsu T, Hachioji M, Saiga K, Takata N, Yokoyama Y, Ozaki T. 2010. Anterior cruciate ligament-derived cells have high chondrogenic potential. Biochem Biophys Res Commun. 391(1): 1142-7.

Gan L, Liao S, Xing Y, Deng S. 2020. The Regulatory Functions of lncRNAs on Angiogenesis Following Ischemic Stroke. Front Mol Neurosci. 13: 613976.

Georgiev GP, Kotov G, Iliev A, Slavchev S, Ovtscharoff W, Landzhov B. 2019. A comparative study of the epiligament of the medial collateral and the anterior cruciate ligament in the human knee. Immunohistochemical analysis of collagen type I and $\mathrm{V}$ and procollagen type III. Ann Anat. 224: 88-96.

Georgiev GP, Landzhov B, Kotov G, Slavchev SA, Iliev A. 2018. Matrix Metalloproteinase-2 and -9 Expression in the Epiligament of the Medial Collateral and Anterior Cruciate Ligament in Human Knees: A Comparative Study. Cureus. 10(11): e3550.

Guo D, Yu H, Huang B, Gao X, Qin Y, Liu X. 2019. Avulsion of the femoral attachment of the medial collateral ligament in the setting of knee multiligament injury: A case report. Medicine (Baltimore). 98(50): e18376.

Hong JY, Shin MH, Douglas IS, et al. 2016. Inhibition of EphA2/EphrinA1 signal attenuates lipopolysaccharide-induced lung injury. Clin Sci (Lond). 130(21): 1993-2003.

Iwakura Y, Ishigame H, Saijo S, Nakae S. 2011. Functional specialization of interleukin-17 family members. Immunity. 34(2): $149-62$.

Jeffries KA, Krupenko NI. 2018. Ceramide Signaling and p53 Pathways. Adv Cancer Res. 140: 191-215.

Jia ZY, Zhang C, Cao SQ, et al. 2017. Comparison of artificial graft versus autograft in anterior cruciate ligament reconstruction: a meta-analysis. BMC Musculoskelet Disord. 18(1): 309.

Kaplan N, Fatima A, Peng H, Bryar PJ, Lavker RM, Getsios S. 2012. EphA2/Ephrin-A1 signaling complexes restrict corneal epithelial cell migration. Invest Ophthalmol Vis Sci. 53(2): 936-45.

Katoh M. 2009. FGFR2 abnormalities underlie a spectrum of bone, skin, and cancer pathologies. J Invest Dermatol. 129(8): 18617.

Kopp F. 2019. Molecular functions and biological roles of long non-coding RNAs in human physiology and disease. J Gene Med. 21(8): e3104.

Koumakis E, Millet-Botti J, Benna JE, et al. 2019. Novel function of PiT1/SLC20A1 in LPS-related inflammation and wound healing. Sci Rep. 9(1): 1808.

Kurogane Y, Miyata M, Kubo Y, et al. 2012. FGD5 mediates proangiogenic action of vascular endothelial growth factor in human vascular endothelial cells. Arterioscler Thromb Vasc Biol. 32(4): 988-96.

LeBert DC, Squirrell JM, Rindy J, et al. 2015. Matrix metalloproteinase 9 modulates collagen matrices and wound repair. Development. 142(12): 2136-46.

Leong NL, Kator JL, Clemens TL, James A, Enamoto-Iwamoto M, Jiang J. 2020. Tendon and Ligament Healing and Current Approaches to Tendon and Ligament Regeneration. J Orthop Res. 38(1): 7-12.

Li J, Cao F, Yin HL, et al. 2020. Ferroptosis: past, present and future. Cell Death Dis. 11(2): 88.

Lin J, Niimi Y, Clausi MG, Kanal HD, Levison SW. 2020. Neuroregenerative and protective functions of Leukemia Inhibitory

Peer) reviewing PDF | (2021:08:65063:1:0:NEW 22 Nov 2021) 
$472 \quad$ Factor in perinatal hypoxic-ischemic brain injury. Exp Neurol. 330: 113324.

473 Lipman K, Wang C, Ting K, Soo C, Zheng Z. 2018. Tendinopathy: injury, repair, and current exploration. Drug Des Devel Ther. 474 12: 591-603.

475 Lucero HA, Kagan HM. 2006. Lysyl oxidase: an oxidative enzyme and effector of cell function. Cell Mol Life Sci. 63(19-20): 2304-16.

Lyon RM, Akeson WH, Amiel D, Kitabayashi LR, Woo SL. 1991. Ultrastructural differences between the cells of the medical collateral and the anterior cruciate ligaments. Clin Orthop Relat Res. (272): 279-86.

Metcalf D. 2003. The unsolved enigmas of leukemia inhibitory factor. Stem Cells. 21(1): 5-14.

Mousavi K, Zare H, Dell\&\#39, et al. 2013. eRNAs promote transcription by establishing chromatin accessibility at defined genomic loci. Mol Cell. 51(5): 606-17.

Nyström A, Velati D, Mittapalli VR, Fritsch A, Kern JS, Bruckner-Tuderman L. 2013. Collagen VII plays a dual role in wound healing. J Clin Invest. 123(8): 3498-509.

Rösler HP. 1979. Unser Bild. Melchior Adam Weikard, Kupferstichsilhouette, H. 23,8 cm, B. 18,2 cm. Künstler und Stecher sind unbekannt. Das Original befindet sich im Stadtarchiv Mainz. Medizinhist J. 14(1-2): 137-41.

Salmena L, Poliseno L, Tay Y, Kats L, Pandolfi PP. 2011. A ceRNA hypothesis: the Rosetta Stone of a hidden RNA language. Cell. 146(3): 353-8.

Santos GC, Silva DN, Fortuna V, et al. 2020. Leukemia Inhibitory Factor (LIF) Overexpression Increases the Angiogenic Potential of Bone Marrow Mesenchymal Stem/Stromal Cells. Front Cell Dev Biol. 8: 778.

Thomopoulos S, Parks WC, Rifkin DB, Derwin KA. 2015. Mechanisms of tendon injury and repair. J Orthop Res. 33(6): 832-9.

Toiyama Y, Okugawa Y, Goel A. 2014. DNA methylation and microRNA biomarkers for noninvasive detection of gastric and colorectal cancer. Biochem Biophys Res Commun. 455(1-2): 43-57.

Verslegers M, Lemmens K, Van Hove I, Moons L. 2013. Matrix metalloproteinase-2 and -9 as promising benefactors in development, plasticity and repair of the nervous system. Prog Neurobiol. 105: 60-78.

Wang C, Chi Q, Sha Y, et al. 2020. Mechanical injury and IL-1 $\beta$ regulated LOXs and MMP-1, 2, 3 expression in ACL fibroblasts co-cultured with synoviocytes. Biotechnol Lett. 42(8): 1567-1579.

Wang C, Sha Y, Wang S, et al. 2020. Lysyl oxidase suppresses the inflammatory response in anterior cruciate ligament fibroblasts and promotes tissue regeneration by targeting myotrophin via the nuclear factor-kappa B pathway. J Tissue Eng Regen Med. 14(8): 1063-1076.

Wang LJ, Zeng N, Yan ZP, Li JT, Ni GX. 2020. Post-traumatic osteoarthritis following ACL injury. Arthritis Res Ther. $22(1)$ : 57.

Wang Z, Wang D, Guo S, Zhuo Q, Jiang D, Yu Z. 2021. Long noncoding RNA distal-less homeobox 2 antisense 1 restrains inflammatory response and apoptosis of periodontal ligament cells by binding with microRNA-330-3p to regulate Ro60, Y RNA binding protein. Arch Oral Biol. 133: 105298.

Xiao K, Yang Y, Bian Y, et al. 2019. Identification of differentially expressed long noncoding RNAs in human knee osteoarthritis. J Cell Biochem. 120(3): 4620-4633.

Xie J, Huang W, Jiang J, et al. 2013. Differential expressions of lysyl oxidase family in ACL and MCL fibroblasts after mechanical injury. Injury. 44(7): 893-900.

Xie J, Jiang J, Huang W, et al. 2014. TNF- $\alpha$ induced down-regulation of lysyl oxidase family in anterior cruciate ligament and medial collateral ligament fibroblasts. Knee. 21(1): 47-53.

Xie J, Wang C, Yin L, Xu C, Zhang Y, Sung KL. 2013. Interleukin-1 beta influences on lysyl oxidases and matrix metalloproteinases profile of injured anterior cruciate ligament and medial collateral ligament fibroblasts. Int Orthop. 37(3): 495-505.

Xie J, Wang CL, Yang W, et al. 2018. Modulation of MMP-2 and MMP-9 through connected pathways and growth factors is critical for extracellular matrix balance of intra-articular ligaments. J Tissue Eng Regen Med. 12(1): e550-e565.

Peer] reviewing PDF | (2021:08:65063:1:0:NEW 22 Nov 2021) 
515 Xin P, Xu X, Deng C, et al. 2020. The role of JAK/STAT signaling pathway and its inhibitors in diseases. Int Immunopharmacol. 516 80: 106210.

517 Yoshida M, Fujii K. 1999. Differences in cellular properties and responses to growth factors between human ACL and MCL cells. $518 \quad$ J Orthop Sci. 4(4): 293-8.

519 Zhang J, Pan T, Im HJ, Fu FH, Wang JH. 2011. Differential properties of human ACL and MCL stem cells may be responsible for 520 their differential healing capacity. BMC Med. 9: 68.

521 Zhang W, Wu Y, Chen H, Yu D, Zhao J, Chen J. 2021. Neuroprotective effects of SOX5 against ischemic stroke by regulating VEGF/PI3K/AKT pathway. Gene. 767: 145148.

Zhang Y, Jiang J, Xie J, et al. 2017. Combined effects of tumor necrosis factor- $\alpha$ and interleukin-1 $\beta$ on lysyl oxidase and matrix metalloproteinase expression in human knee synovial fibroblasts in vitro. Exp Ther Med. 14(6): 5258-5266.

525 Zhou M, Hu H, Han Y, et al. 2021. Long non-coding RNA 01126 promotes periodontitis pathogenesis of human periodontal ligament cells via miR-518a-5p/HIF-1 $\alpha$ /MAPK pathway. Cell Prolif. 54(1): e12957.

527 Zhou N, Zhao WD, Liu DX, et al. 2011. Inactivation of EphA2 promotes tight junction formation and impairs angiogenesis in brain endothelial cells. Microvasc Res. 82(2): 113-21. 
Figure 1

Comparative analysis of DEGs in the normal and injured groups of ACL

A: Volcano plot of the differentially expressed mRNAs. The red and green dots represent statistically significantly up-regulated and down-regulated mRNAs.B:Hierarchical clustering shows a difference in mRNA expression profile between the two groups and homogeneity within groups. C-E: Top 20 highest enriched GO terms for differentially expressed mRNAs. F: Top 20 highest enriched KEGG pathways for differentially expressed mRNAs.
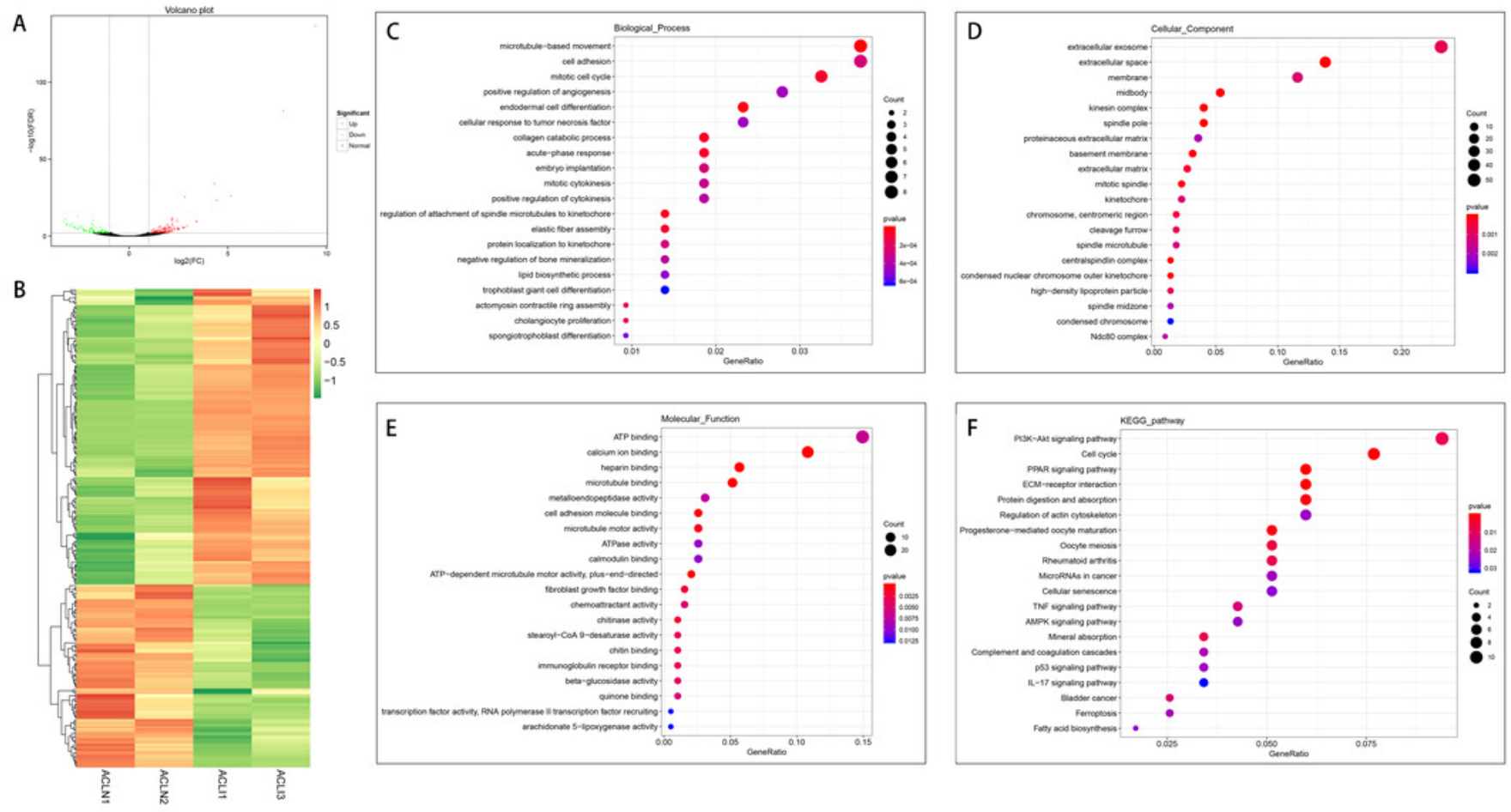
Figure 2

Comparative analysis of DEGs in the normal and injured groups of MCL

A: Volcano plot of the differentially expressed mRNAs. The red and green dots represent statistically significantly up-regulated and down-regulated mRNAs.B:Hierarchical clustering shows a difference in mRNA expression profile between the two groups and homogeneity within groups. C-E: Top 20 highest enriched GO terms for differentially expressed mRNAs. F: Top 20 highest enriched KEGG pathways for differentially expressed mRNAs.

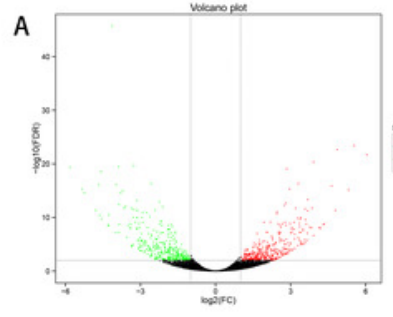

B

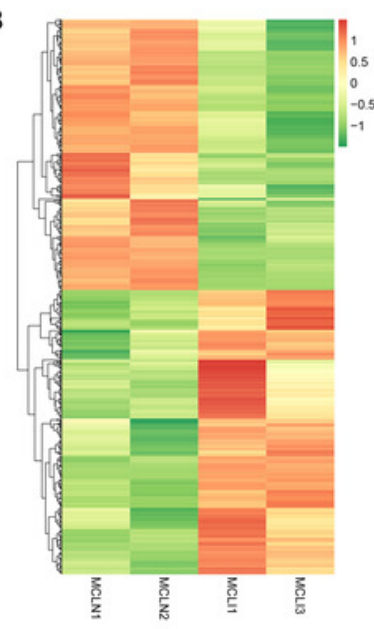

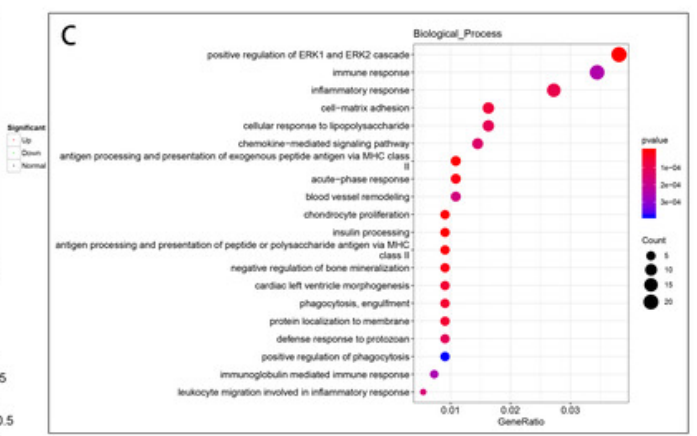
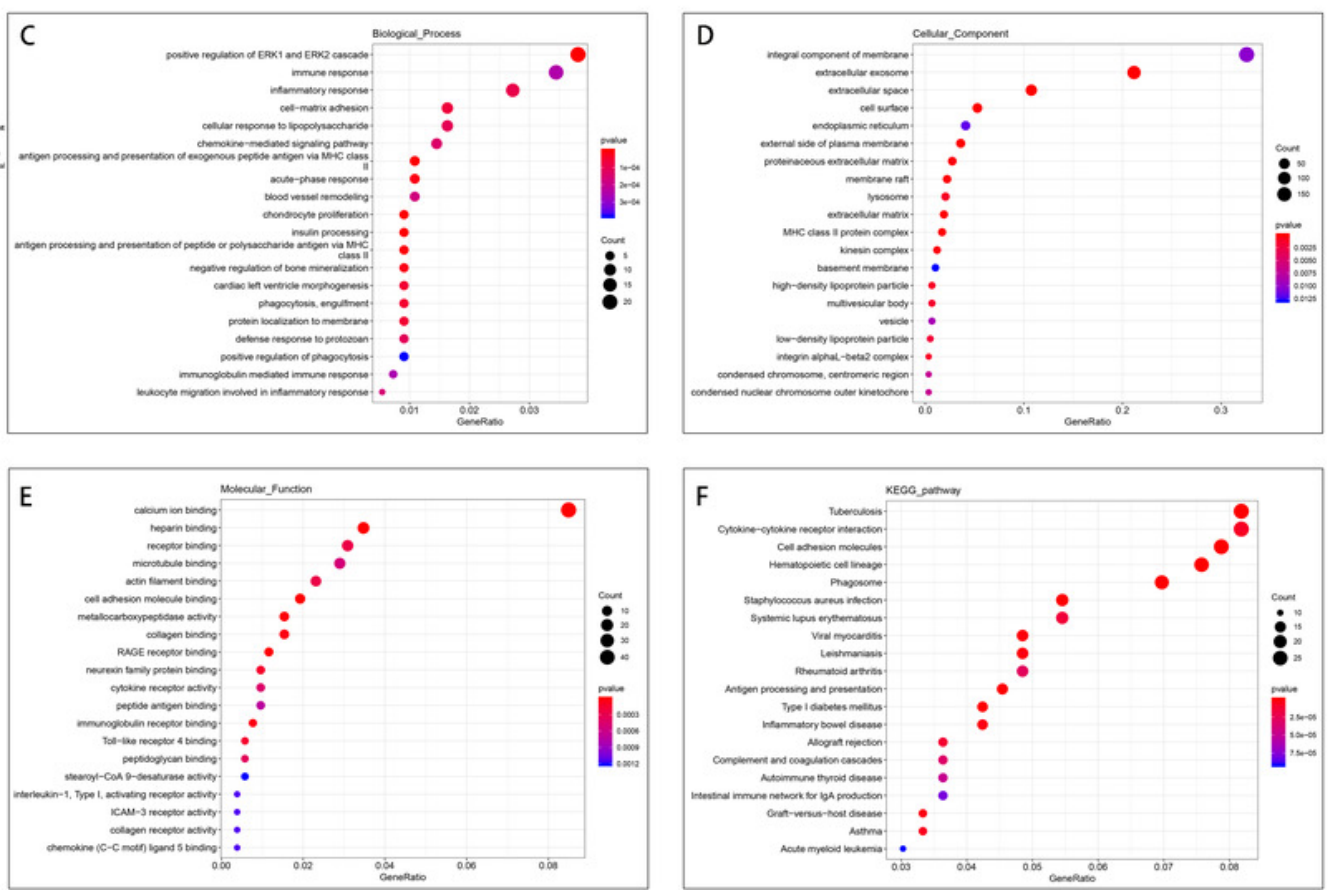
Figure 3

Comparative analysis of DEGs in $\mathrm{ACL}$ and $\mathrm{MCL}$ of the normal group

A: Volcano plot of the differentially expressed mRNAs. The red and green dots represent statistically significantly up-regulated and down-regulated mRNAs.B:Hierarchical clustering shows a difference in mRNA expression profile between the two groups and homogeneity within groups. C-E: Top 20 highest enriched GO terms for differentially expressed mRNAs. F: Top 20 highest enriched KEGG pathways for differentially expressed mRNAs.
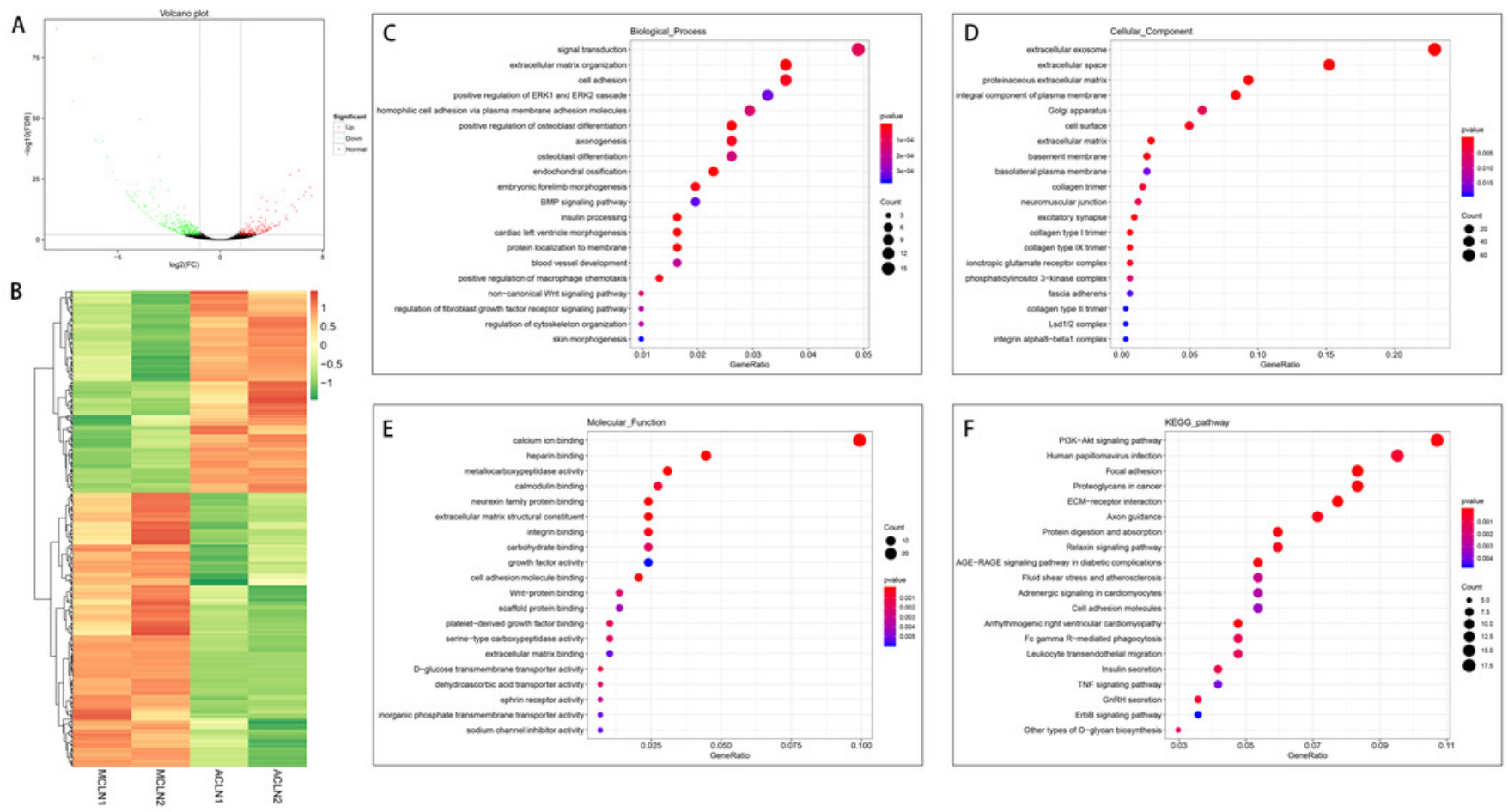
Figure 4

Comparative analysis of DEGs in ACL and MCL of the injured group

A: Volcano plot of the differentially expressed mRNAs. The red and green dots represent statistically significantly up-regulated and down-regulated mRNAs.B:Hierarchical clustering shows a difference in mRNA expression profile between the two groups and homogeneity within groups. C-E: Top 20 highest enriched GO terms for differentially expressed mRNAs. F: Top 20 highest enriched KEGG pathways for differentially expressed mRNAs.
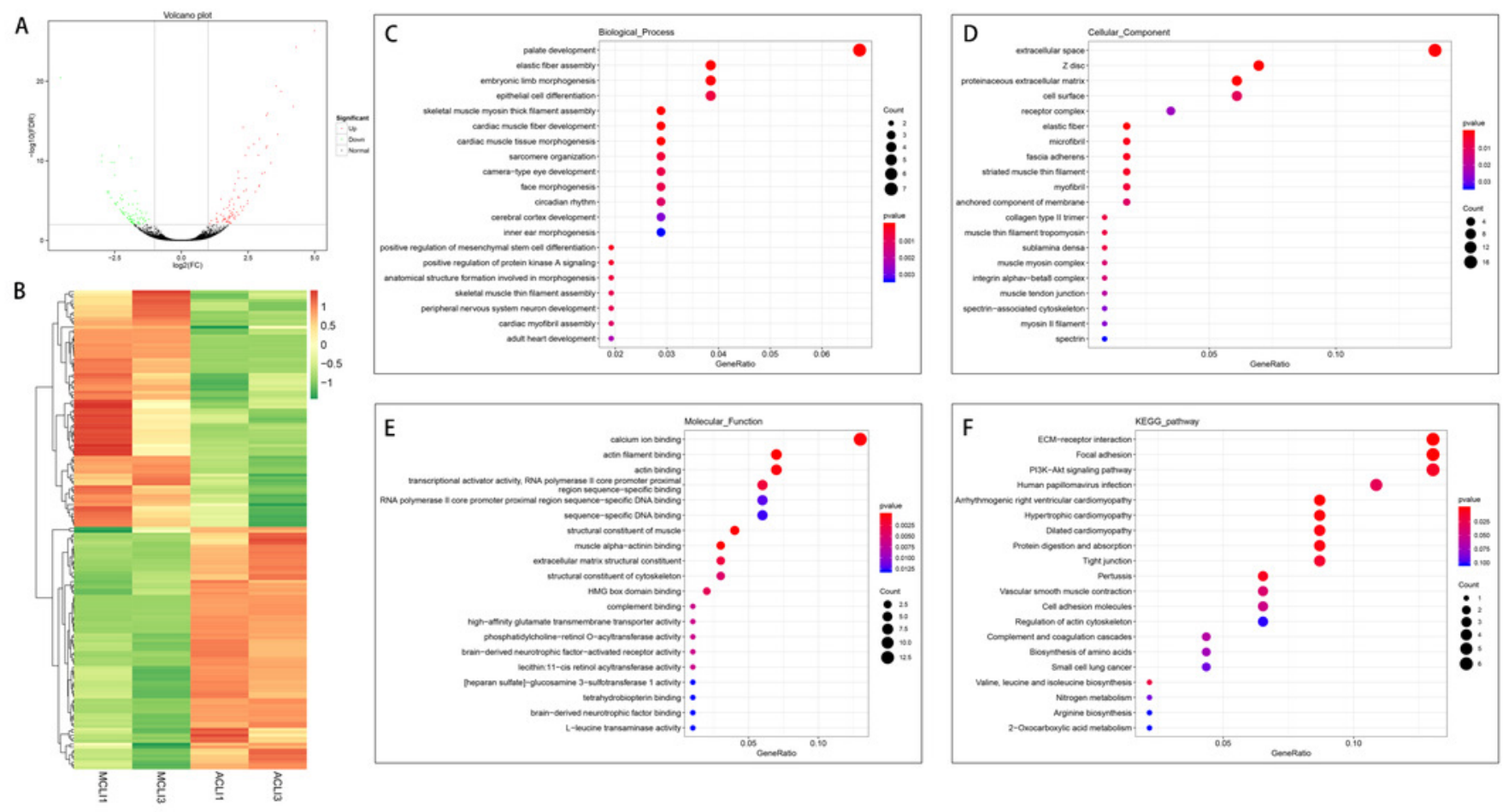
Figure 5

Validation of DEGs and DELs by qRT-PCR

A-B: normal group vs. injured group A: ACL, B: MCL. C-D: MCL vs. ACL. C: normal group, D: injured group. The $\mathrm{x}$-axis represents the gene name, and the $\mathrm{Y}$-axis represents the relative expression of DEGs and DELs. *Significantly expressed DEGs and DELS

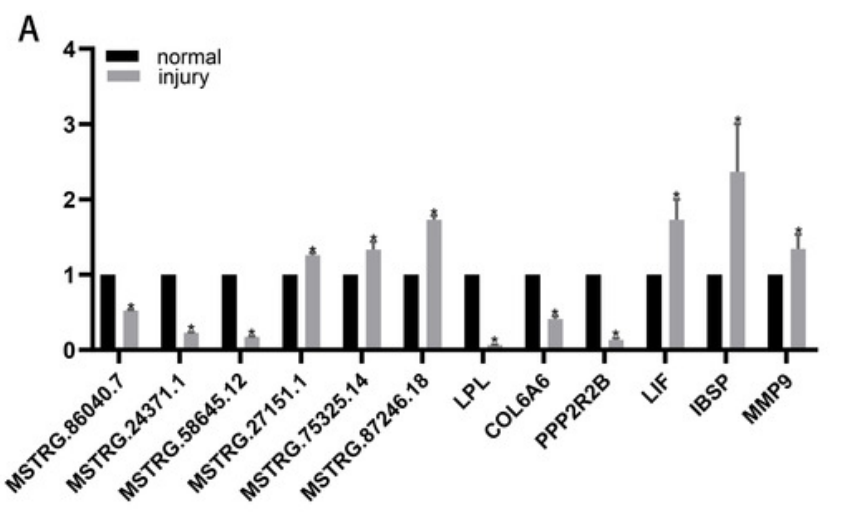

ACLNvsACLI

C

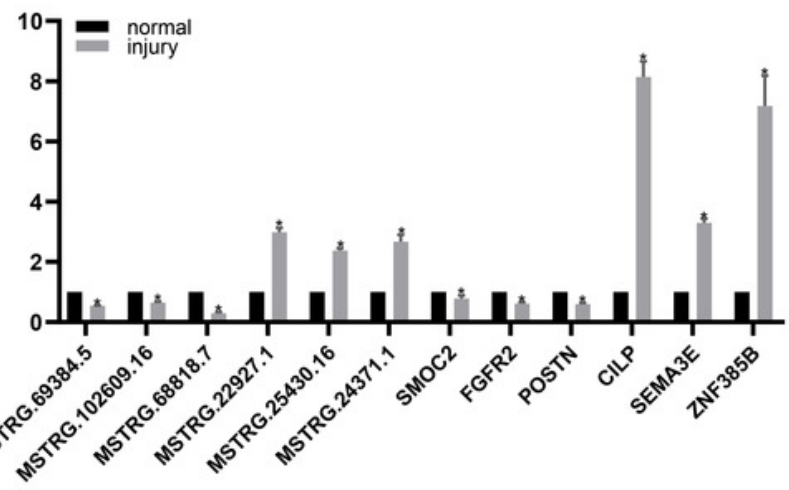

MCLNvSACLN

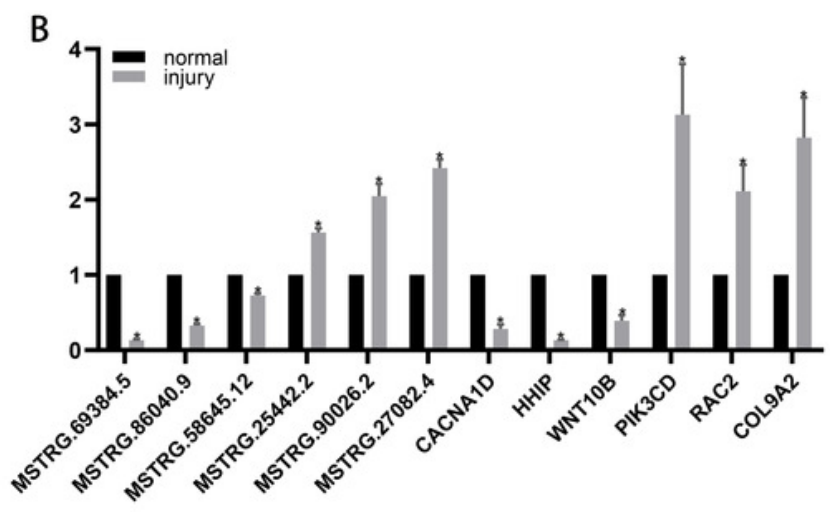

MCLNvsMCLI

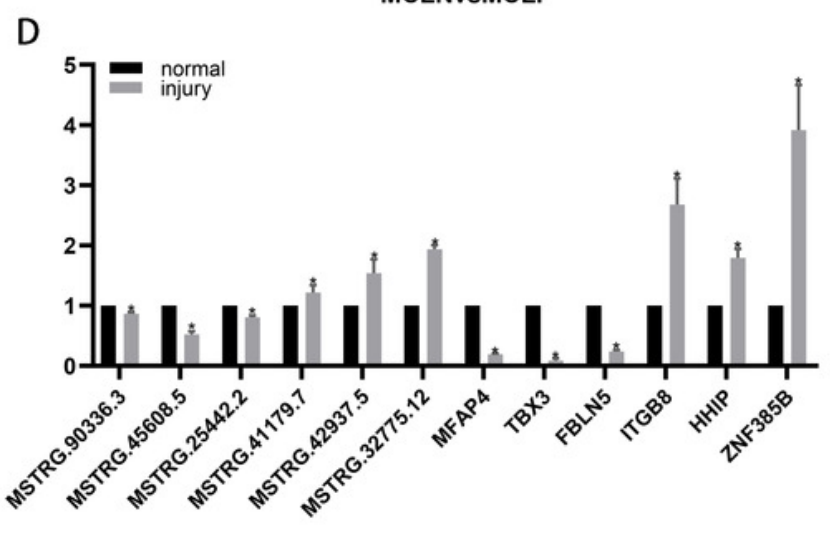

MCLIvsACLI 


\section{Figure 6}

LncRNA-miRNA-mRNA ceRNA regulation network in each group(the top 50 nodes).

LncRNAs, miRNAs, and mRNAs are represented by arrows, diamonds, and circles, and upand down-regulation are represented by red and green. A-B: normal group vs. injured group. A: ACL, B: MCL. C-D: MCL vs. ACL. C: normal group, D: injured group. E-F: Regulatory network of COL7A1 and LIF. E: ACL F: MCL. G: Regulatory network of FGD5, SLC20A1

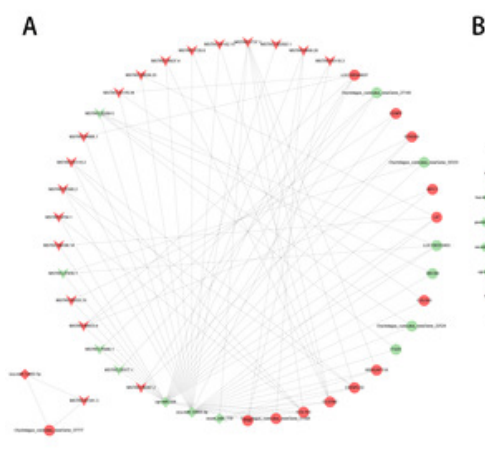

B

E

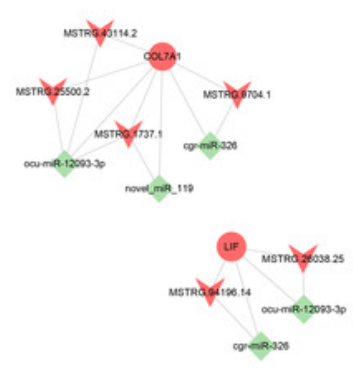

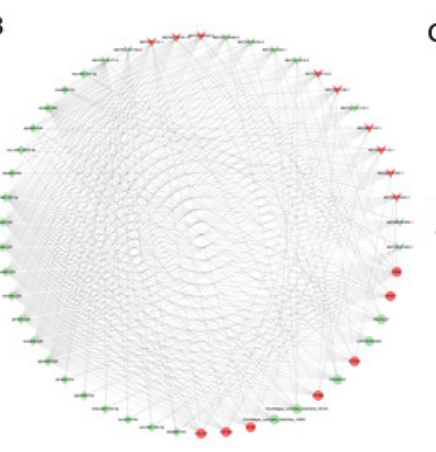

F

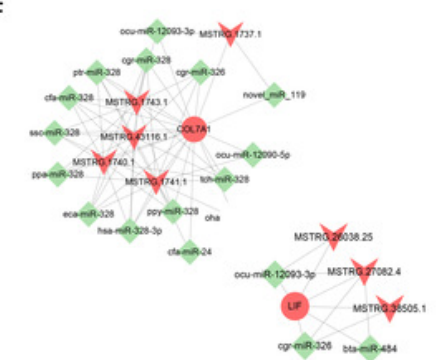

C

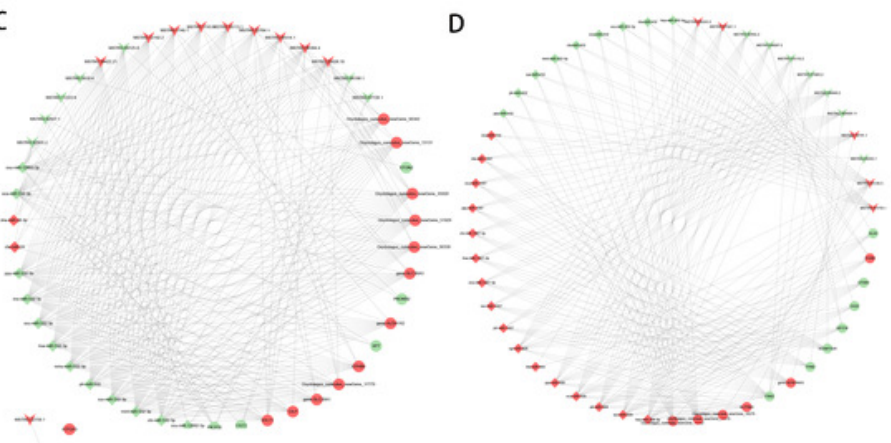

G

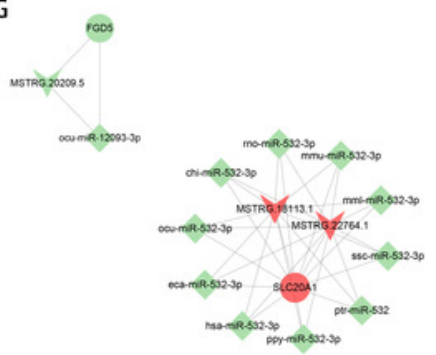


Figure 7

PPI network analysis in each group of DEGs (top 30 hub genes).

The higher score has a red color, and the lower score has a yellow color. A-B: normal group vs. injured group. A: ACL, B: MCL. C-D: MCL vs. ACL. C: normal group, D: injured group.
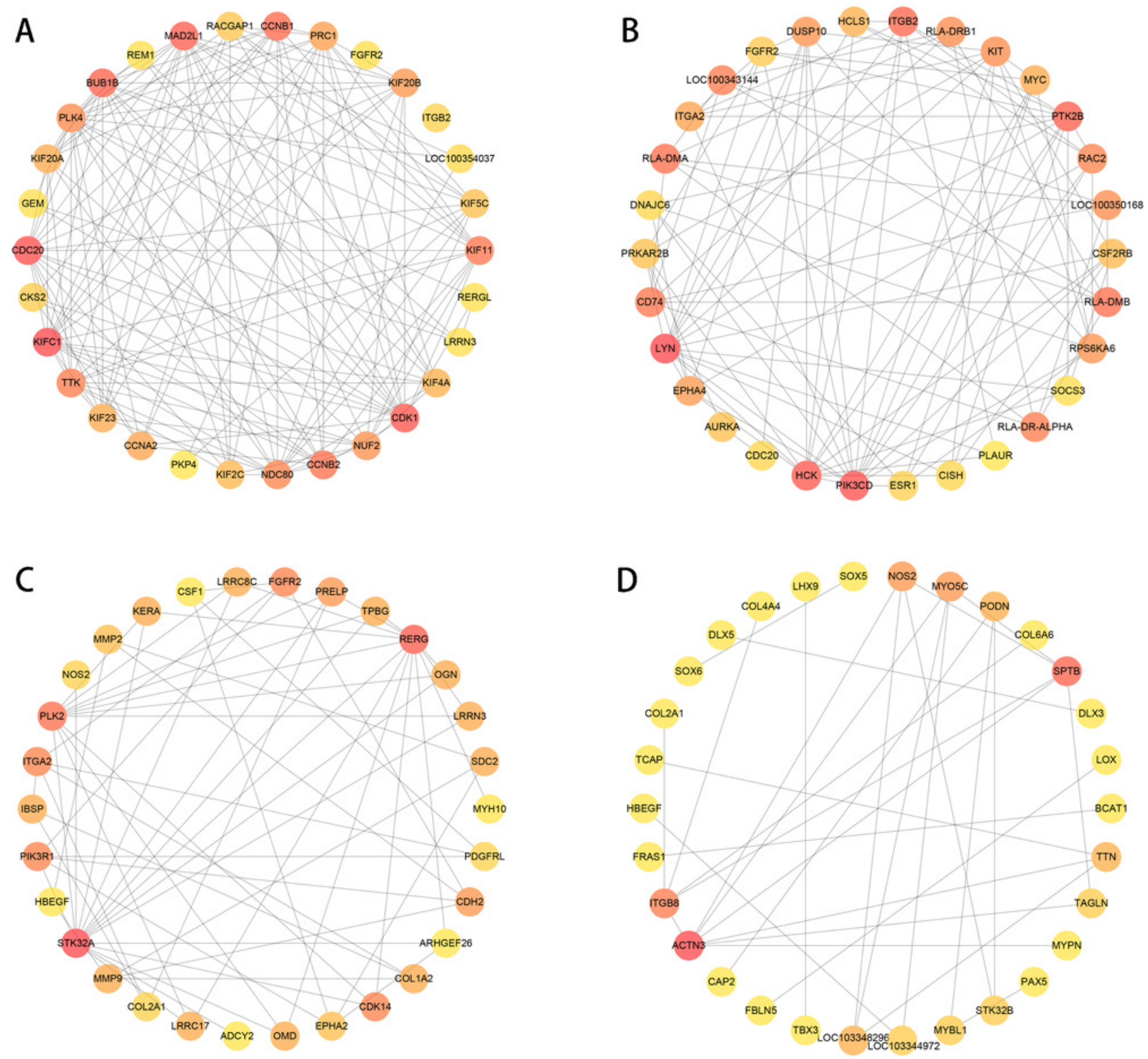